\title{
La apropiación de la biomúsica \\ por el cuidador primario de niños \\ con el síndrome de Williams
}

M. en C. Julio Hernández Falcón * y Biól. Víctor Valverde Molina*.

*Megaproyecto "Nuevas Estrategias Genómicas y Proteómicas en Salud Pública" SDEI. PTID.05. 1 y ENEO-UNAM

* Adscritos a la Unidad de Investigación y Posgrado ENEO-UNAM.

Enseñar y motivar al cuidador primario de niños con diagnóstico de Síndrome de Williams, a conocer su cuerpo y a cuidarlo fue nuestro principal objetivo, ya que adolecen de herramientas para afrontar el desgaste y estrés. Nuestro abordaje se sustentó en la investigación-acción emprendida por grupos o comunidades con la finalidad de cambiar su situación de vida.

La acción se orientó hacia la habilitación de los padres, en la conducción de la biomúsica. En el estudio se diseñaron instrumentos de autoevaluación de la expresión corporal.

Los resultados muestran efectos inmediatos de la musicoterapia sobre la expresión corporal manifiestos en la disminución de la resistencia muscular y de las cifras tensionales, así como diversos indicadores de una percepción corporal congruente con la salud.

Palabras Clave: Biomúsica, Cuidador Primario, Musicoterapia, Síndrome de Williams.

\section{Abstract}

To teach and to motivate to the primary caregiver of children with diagnosis of Syndrome of Williams, to know its body and to take care of it was our main objective, since they suffer from tools to confront the wearing down and stress. Our boarding sustained in the investigation-action undertaken by groups or communities with the intention of changing its situation of life.

The action was oriented towards the rating of the parents, in the conduction of the bio-music. In the study instruments of self-evaluation of the corporal expression were designed.

The results show immediately effects of the music therapy under the corporal expression manifest in the diminution of the muscular resistance and of the cipher of blood pressure, just as diverse manifestation sing corporal perception congruent with the health.

Key words: Biomusic, Primary Caregiver, Music therapy, Williams Syndrome. 


\section{INTRODUCCION}

El síndrome de Williams al igual que muchos de los defectos al nacimiento, representan para los cuidadores primarios un evento critico, ya que impone demandas de atención que no se pueden cubrir sin modificar y ajustar los roles familiares. De igual manera que en los casos de carácter crónico, el Síndrome de Williams demanda atención por parte del profesional de Enfermería que, establece una relación de ayuda basada en la autenticidad, la sensibilidad y la empatía. Pero sobre todo, un profesional de Enfermería atento y abierto a la gestión de un cuidado complementario que desde un enfoque preventivo y terapéutico incida sobre las necesidades presentes y potenciales del Cuidador Primario.

Por su parte, el cuidador primario confronta una situación donde el propio rol se ve comprometido ante las fantasías y falsas expectativas de recuperación; lo que representa una amenaza no solo para la continuidad del cuidado sino para la constitución de una experiencia creativa de la crisis que se confronta. La impotencia de los cuidadores primarios no sólo deriva de la cronicidad del problema sino del debilitamiento muchas veces coexistente de las redes sociales de apoyo social y familiar; sin olvidar que la propia experiencia demanda el desarrollo de competencias de cuidado solo posibles a través de un proceso de aprendizaje.

Ante este panorama y tomando en cuenta la valoración Enfermera de los Cuidadores Primarios de niños con Síndrome de Williams, se hace necesario promover una intervención cuyo propósito central sea inducir en los padres la esperanza, la motivación, la relajación, la recuperación creativa, a través de la promoción y utilización de la Biomúsica.

\section{OBJETIVO}

Desarrollar las habilidades del cuidador primario de niños con diagnóstico de Síndrome de Williams para afrontar el desgaste y estrés del propio rol a través de la Biomúsica.

\section{MARCO TEÓRICO}

\section{Cuidador Primario}

Sabemos que en nuestro medio familiar se cuida con dedicación y afecto. Sin embargo quienes cuidan, no siempre están preparados para responder a esta tarea que incluye; tensión, esfuerzo e impacto emocional.

En la mayoría de las familias, un único miembro asume la mayor responsabilidad; generalmente una mujer: esposa hija, hermana. Muchas veces, sin que haya un acuerdo explícito. Habitualmente también se sigue atendiendo las necesidades del resto de la familia. Por lo que es necesario el decidir dónde y cuándo invertir esfuerzos y energías.

Simultáneamente se pueden dar sentimientos de satisfacción por contribuir al bienestar así como negativos que incluyen: la sensación de impotencia, culpabilidad, soledad, preocupación, tristeza y cansancio. Incluso puede haber consecuencias laborales, aumentar la sensación de incumplimiento en el trabajo o el cuidado de la familia. Además de la reducción de las actividades sociales y aparecen sentimientos de aislamiento del mundo que nos rodea'.

En ocasiones suele haber problemas de salud por la presión emocional, las relaciones familiares tensas, los sentimientos de impotencia, tristeza o censura. Son difíciles de señalar porque dependen de cada familia y del familiar que se cuida. Sin embargo, existe una constante que es característica del cuidador: el que cuida se exige más allá de sus posibilidades, llegando incluso a perjudicarse como lo reflejan estados de inmunosupresión².

Lo importante es que el cuidador primario identifique las manifestaciones desequilibrantes: correr a todas partes, no tener tiempo, el perder el sueño, la fatiga, el aislamiento, los problemas físicos, los trastornos digestivos, el dolor de cabeza, los trastornos de la memoria, la dificultad para concentrase. Los sentimientos de depresión o nerviosismo y sobre todo la no aceptación de las manifestaciones físicas o psicológicas que lo pueden conducir de cuidador funcional a uno de supervisor que procura solo los cuidados elementales ${ }^{3}$.

\section{Síndrome de Williams}

Es la delección del cromosoma 7 que afecta a 16 genes y se caracteriza por: retraso en el desarrollo, retraso mental leve o moderado; trastorno del desarrollo articular y daño cardiovascular. Físicamente se trata de niños con labios prominentes y boca semi-abierta, dientes pequeños y separados, iris del ojo estrellado y problemas visuales. De personalidad locuaz y muy sociable; ansiosos y emotivos. Pueden presentar depresión y están muy pendientes de la personalidad de los demás; así como de sus estados de ánimo. Sin embargo, poseen memoria auditiva por lo tanto mayor habilidad para la música ${ }^{4}$.

\section{Biomúsica}

A la descorporización, la podemos definir como no estar en el propio cuerpo, esta situación se presenta en la enfermedad tanto física como psicológica. Subjetivamente 
se experimenta como una ausencia de la experiencia del ser que lleva a estados depresivos y a la compasión hacia sí mismo.

Las técnicas de recorporización incrementan la percepción del cuerpo, tal es el efecto del masaje, el Rolfing, la retroalimentación biológica, la músicoterapia ${ }^{5}$. Ginger Clarkson destaca el valor de la musicoterapia a través de su experiencia de más de 25 años de trabajo con personas de distintas edades y que han trascendido la ansiedad, el dolor y la frustración a través de la música ${ }^{6}$.

La Biomúsica o también llamada Musicoterapia Evolutiva (MTE) representa un complejo de técnicas que integra: el juego cooperativo7, la música, el canto, la libre expresión, la creatividad, el ejercicio respiratorio, la narración y la fantasía guiada.

Por su sencillez la MTE representa una alternativa viable en el entrenamiento de los cuidadores primarios que confrontan crisis. La música puede equilibrar porque el sonido y el ritmo correctamente dirigidos pueden hacer resonar el cuerpo o la parte del cuerpo que nos interesa. Cada parte del cuerpo de acuerdo a su constitución vibra a una determinada frecuencia, recibe y emite vibraciones. La música actúa física y psicológicamente ${ }^{8}$.

"Llamamos a Biomúsica la Musicoterapia Evolutiva (MTE) porque consideramos que una persona (más allá de una patología declarada o de una aparente normalidad) es un ser en evolución permanente, dinámico, en cambio constante9 .

La música influye sobre el ritmo respiratorio, la presión arterial, las contracciones estomacales y los niveles hormonales. Los ritmos cardiacos se aceleran o se vuelven más lentos de forma tal que se sincronizan con los ritmos musicales. Disminuyen los niveles de corticoides, aumentan los niveles de endorfinas, de la interleucina 1 así como los niveles de lg A y lg G. Alivia la depresión y favorece la autoestima. También se sabe que la música puede alterar los ritmos eléctricos de nuestro cerebro $10,11,12$

\section{METOdOLOGÍA}

Por su carácter de investigación-acción se convocó a los padres (11) de la Asociación Nacional de Niños con Síndrome de Williams A.C. (ANNSWAC) a participar en el desarrollo de competencias de cuidado y a partir de las necesidades detectadas por el equipo de investigadores Niveles de estrés evaluado a través del dibujo de la figura humana y de la autoevaluación de la expresión corporal.

\section{Investigación acción}

Integra tanto la investigación como producción de conocimientos y la acción concreta para modificar de manera intencional la realidad. En la investigación acción se construye la experiencia educativa donde "todos aprendemos de todos". Tiene por objetivo principal, la liberación del potencial creador de la comunidad, así como la movilización de los recursos humanos para la solución de los problemas sociales.

\section{Sesiones semanales de Biomúsica.}

En el periodo de octubre 2006 a abril 2007, se realizaron sesiones de dos horas por semana en las instalaciones de la Casa Coahuila. El material de apoyo incluyó equipo de sonido y cintas gravadas.

Las actividades de biomúsica se estructuraron con base a la metodología explicitada en el libro de Mario Corradini que incluye:

Ludicidad: juegos cooperativos.

Ejercicios de Biomúsica: las Intervenciones fueron acompañadas por música diseñada para tales fines (Mario Corradini, autor) ${ }^{13}$, que comprenden diferentes momentos acoplados de la siguiente manera:

1. Ejercicio de relajamiento y alineación de la columna vertebral (Pista 7).

2. Ejercicio para favorecer la tensión-relajación de la musculatura corporal (Pista 7).

3. Ejercicios acompañados de una respiración rítmica, una visualización y emisión de sonidos dirigidos por los participantes a zonas específicas del cuerpo, uno por sesión (Pista 1 a la 5).

4. Cierre de la sesión con ejercicios diseñados para un equilibrio psicofísico (Pista 6).

Para el seguimiento y evaluación del taller por los propios participantes se diseño un instrumento de valoración que incluyó: el lenguaje corporal y la libre asociación. Desde el punto de vista fisiológico se incluye la valoración de las cifras de tensión arterial antes del inicio de la sesión y posterior a su realización. Así mismo se considero una valoración inicial de los participantes a través del dibujo de la figura humana para determinar el nivel de estrés de los participantes y poder establecer estrategias de ayuda y manejo individualizado. La muestra tiene un carácter no estadístico y a conveniencia.

Durante la realización del taller los participantes emitieron su juicio y su registro se plasmó en cinta magnética: grabación de entrevistas 


\section{RESULTADOS Y DISCUSION}

El grupo de inicio comprendió 11 padres de los cuales 3 salieron por complicaciones de salud de los infantes. Así que sólo 8 de los padres realizaron 25 sesiones ( 5 sesiones para cada sonido dirigido), con intervalos para su valoración.

\section{La valoración inicial}

- Una cuarta parte de los padres se ubicó en menos de 40 años y más de un tercio entre los 40 a 49 años y otro mayor de 50 años.El total de los participantes fueron 6 mujeres y 2 hombres tres cuartas partes casados y el resto solteros o viudos

Resultados del cuestionario clínico para el diagnóstico de síndrome depresivo.

- Una mayoría se ubicó con normalidad y una cuarta parte manifestó reacción de ansiedad y en un sólo caso se encontró depresión incipiente.

A través del dibujo de la figura humana realizado por los participantes al inicio del programa se pudo establecer la necesidad de atención especializada en solo cuatro de los casos que revelaron niveles de estrés elevado. El instrumento de valoración de la actitud corporal aplicado por los participantes al inicio de la sesión y al final de esta reveló no solo la necesidad de ser más conscientes del propio cuerpo y de las manifestaciones del mismo frente a los problemas de la vida cotidiana sino la propia limitación para hacer un proceso consciente autodirigido, es decir se evidenció la resistencia para hacer consciente los procesos reflejados en la expresión corporal. Sin embargo, las resistencias poco a poco se fueron venciendo entre los participantes hasta hacer de la valoración un proceso rutinario.

\section{Cambios en la expresión corporal}

Los efectos de la Biomúsica se manifestó en: rostro feliz; disminución de la resistencia muscular; equilibrio en las cifras tensionales; disminución del malestar general; disminución del cansancio; mejora la posición anatómica de la columna; anulación del dolor de cabeza y muscular.

En cada una de las sesiones los comentarios fueron de carácter positivo a pesar del retrazo en el inicio de las sesiones y el involucramiento progresivo de los participantes. El ambiente fue cooperador y motivante para los participantes pese a interrupciones debido a las actividades del lugar (eventos sociales) e incluso algunas sesiones se prolongaron en tiempo. Los participantes no solo fueron los padres sino que también los niños con Síndrome de Williams una vez terminada su clase de canto se incorporaron a la sesión.

\section{Entrevistas}

\section{Antes}

- "Cuando la niña nació y me di cuenta que no era igual...toda la carga la deje en mi esposo".

- "Lamento llevar a cabo el cuidado de mi hija" "Aún me pregunto ipor qué?".

- "Tiene la prospectiva de que tu hijo sea igual que los demás...poco a poco te das cuenta que es diferente esto es frustrante".

- "A veces supera toda posibilidad y le veo sumamente integrada y estimulada".

\section{Después}

- "Nos relajamos y salimos un poquito desestresados".

- "A veces traigo dolor. Yo creo por el mismo estrés que uno trae. $Y$ luego con el ejercicio sale uno adolorido, pero como que las últimas veces termina uno con mucho cansancio pero a la vez tranquila".

- "Me voy a dormir con toda la tranquilidad del mundo".

- "Me integre al grupo y termine con la presión normal y muy estimulada.

Una forma de potenciar directamente la autoestima, la autonomía y la autodeterminación es escuchando, reconociendo y tomando en cuenta el saber respecto la propia salud y sus necesidades, favoreciendo el conocimiento del cuerpo y el autocuidado del mismo.

Los padres han emprendido un camino hacia el autoconocimiento y se están capacitando como promotores del autocuidado para llevar a otras asociaciones la experiencia.

\section{CONCLUSIONES}

En el cuerpos existen un sinnúmero de posibilidades terapéuticas autógenas. Enseñar y motivar a los individuos a conocer su cuerpo y a cuidarlo consiste una tarea prioritaria, que involucra a los profesionales de la salud y a la sociedad en su conjunto. La investigación acción emprendida junto con la ANSWAC es una muestra del valor de la Biomúsica para el manejo no solo del cuerpo como posibilidad terapéutica sino como el desarrollo de alternativas viables para el manejo del estrés de la enfermedad de carácter crónico. Los hallazgos son alentadores y su continuación necesaria en una sociedad que ha menospreciado el cuerpo cuando carece de interés económico. 
Ahora es necesario proseguir esta investigación ya que estudios como los realizados en relación a la biomúsica (Rodríguez.S y col.; 2007) muestran datos consistentes con sus efectos en la corporeidad de los alumnos y su valor terapéutico.

Finalmente queremos destacar la labor contundente de la Enfermería profesional con un enfoque holístico, al procurar los cuidados indispensables para que el cuidador primario pueda desarrollar sus actividades cotidianas, ya que al asegurar la salud del cuidador primario, afianzamos la del paciente.

\section{REFERENCIAS BIBLIOGRÁFICAS}

1. Sánchez-H., B. Habilidad de cuidado de los cuidadores en situación de Enfermedad crónica. En: El arte y la ciencia del cuidado Universidad Nacional de Colombia-2002. pp.373385.

2. Kiecolt-G, JK y col. El estrés psicológico enlentece la cicatrización de las heridas. Lancet (Ed. Esp.) 1996, 28, 188-191.

3. Bayés R, Arranz P, Barbero J, Barreto P. Propuesta de un modelo integral para una intervención terapéutica paliativa. Medicina Paliativa 1996, 3, 114-121.

4.http://www.discapnet.es/Discapnet/Castellano/Salud/Enfermedades/W/Sindrome+de+Williams/Cover+Williams.htm

5. Sydney M. Jourard. La personalidad saludable. Un punto de vista de la psicología humanística. México, Ed. Trillas. 1987.
6. Ginger Clarkson. Soñe que era normal. México, Alfaomega editor. 2000.

7. Brown, G. ¿Qué tal si jugamos otra vez? Lumen/Humanitas. Buenos Aires, Argentina 4ta. Ed. 1998. 146 pp

8. Almanza-M, Pilar. La terapia musical como intervención Enfermera. Enfermeria Global. No. 2. Mayo 2003 http://www. um.es/eglobal/2/02e04.html.

9. Corradini, M. Biomúsica: la musicoterapia evolutiva. Santa Fe, Argentina. 2006. 279 pp.

10. Watkins, Gwendollyn RMS. Music Therapy: Proposed Physiological Mechanism and Clinical Implications. Clinical Nurse Specialist. 1997. March; $11(2): 43-50$.

11. Mizue Suzuki RN y col. Behavioral and endocrinological evaluation of music therapy for elderly patines with dementia. Nursing and Heath Sciencies. 2004. 6: 11-18

12. ZARATE-D, Patricia y DIAZ T, Violeta. Aplicaciones de la musicoterapia en la medicina. Rev. méd. Chile, feb. 2001, vol.129, No.2, p.219-223

13. Mario Corradini, CD distribuído por Biomusica Internacional: www.biomusica.com.

14. Rodriguez S.; Sanchez E.; Valverde M. Impacto de la biomusica en la salud mental de las y los estudiantes de Enfermería. Una experiencia pedagogica. (Cartel presentado en el XVII Congreso Internacional de la FEPEN-2007)

\section{DIRECCIÓN PARA CORRESPONDENCIA}

juliohernandezfalcon@yahoo.com.mx victorvalverde@eneo.unam.m

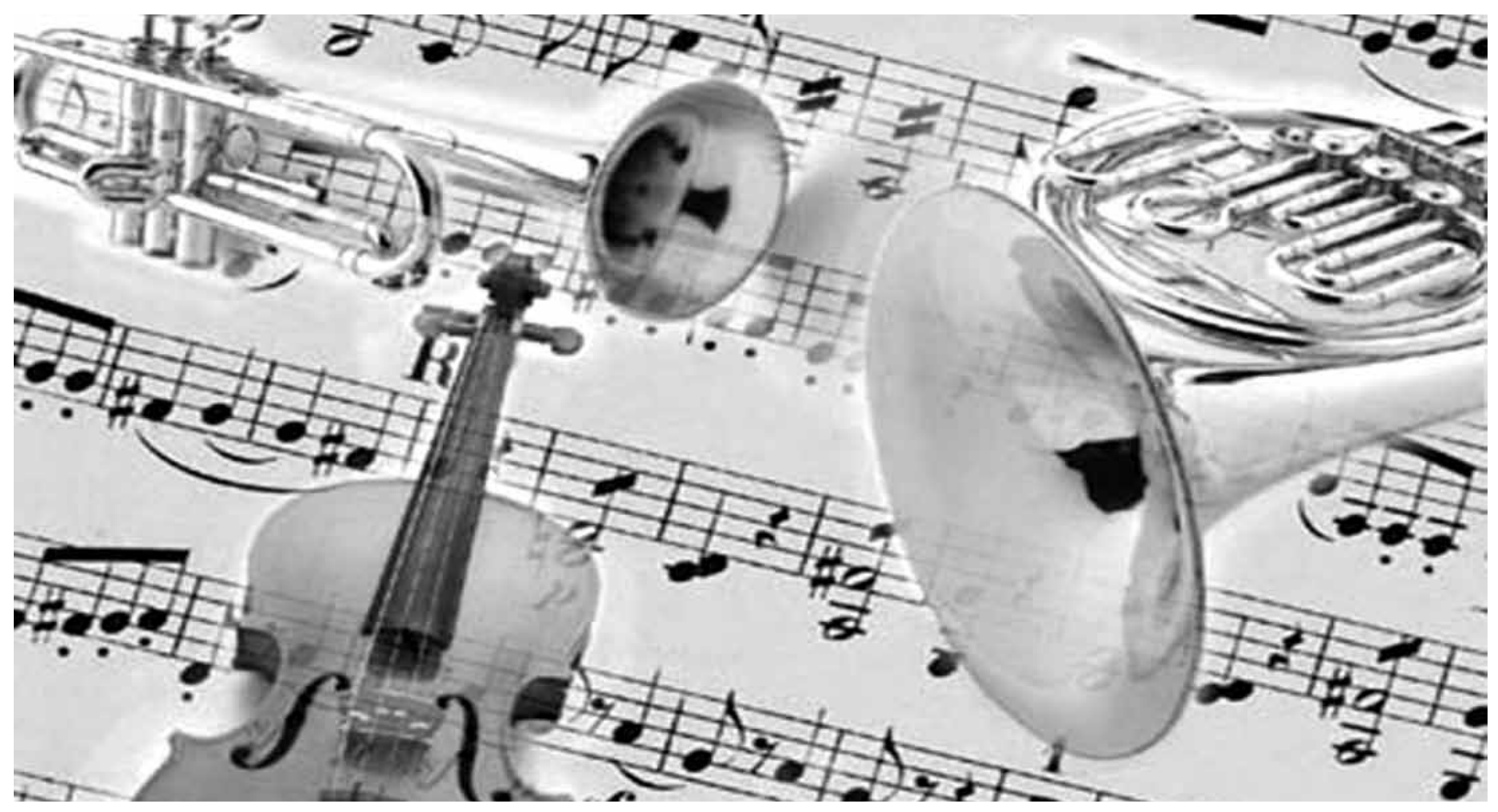

